SHORT REPORT

\title{
Late epilepsy following open surgery for aneurysmal subarachnoid haemorrhage
}

\section{S J Buczacki, P J Kirkpatrick, H M Seeley, P J Hutchinson}

J Neurol Neurosurg Psychiatry 2004;75:1620-1622. doi: 10.1136/jnnp.2003.026856

Objective: To determine the risk for late epilepsy ( $>2$ weeks postoperatively) following aneurysmal subarachnoid haemorrhage (SAH) treated by early aneurysm clipping.

Design: Subgroup analysis of the East Anglian regional audit of SAH (1994-2000; $n=872)$ with 12 month follow up. Prophylactic anticonvulsants were not routinely prescribed unless there was a perioperative seizure.

Subjects: 472 patients with aneurysmal SAH undergoing surgical clipping of the aneurysm were studied. Patients presenting in WFNS grade $V$, with space occupying haematomas requiring emergency surgery, or with posterior circulation aneurysms, rebleeds, and surgery after 21 days were excluded.

Results: Late epilepsy occurred in 23 patients (4.9\%). There was a correlation between the incidence of late epilepsy and both the presenting WFNS grade $(p<0.05)$ (grade 1, 1.4\%; grade 2, 3.8\%; grade 3, 9.6\%; grade 4, 12.5\%) and the Glasgow outcome score at discharge $(p<0.01)$ (good recovery, $2.2 \%$; moderate disability, $5.0 \%$; severe disability, $15.5 \%$ ). There was no relation between the incidence of late epilepsy and sex or the site of the aneurysm.

Conclusions: The low incidence of late epilepsy following open surgery for aneurysmal SAH supports the withholding of prophylactic anticonvulsants. Patients with poor WFNS grade and poor recovery after surgery are at increased risk and should be closely monitored.

$\mathrm{T}$ he true incidence of late epilepsy (more than two weeks postoperatively) after subarachnoid haemorrhage (SAH) is unclear, with quoted figures of between 3\% and 35\%. ${ }^{1-8}$ Independent predictive factors that have been reported include age, presenting clinical grade, ${ }^{9}$ density of haemorrhage, occurrence of rebleeding, and the location of the aneurysm. ${ }^{10}$ Middle cerebral artery aneurysms are thought to show vulnerability to long term epilepsy. The outcome in terms of the Glasgow outcome score (GOS) has been shown to be predictive in some series but not in others. ${ }^{11}$

Since these studies have been published there have been significant advances in the surgical management of subarachnoid haemorrhage, ${ }^{12}$ notably a policy of aggressive resuscitation, early surgery, and improvements in neurointensive care. Consequently, good clinical outcome is now in enjoyed by the majority of patients ${ }^{13}{ }^{14}$ and a reduction in the rate of long term epilepsy is expected. As the efficacy of prophylactic anticonvulsants is uncertain and they are not without side effects, risk stratification of patients with SAH could be useful.

Our objectives in this study were first, to determine the incidence of late epilepsy with modern management of patients with SAH; second, to determine independent
Table 1 Exclusion criteria (from Ross et a ${ }^{15}$ )

- Initial angiogram negative for SAH

- Aneurysms treated endovascularly

- Space occupying haematomas requiring emergency treatment

- Presenting WFNS grade V

- Could not at least flex to pain after resuscitation

- Clinical deterioration before surgery resulting in inability to flex to pain

- Non-aneurysmal SAH

- Bleed from an infratentorial aneurysm

- Rebleed before surgery

- Multiple aneurysms in which the source of the bleed was unclear

- Surgery occurring after 21 days

- Patients who had died during hospital stay or in the year following surgery

SAH, subarachnoid haemorrhage; WFNS, World Federation of Neurosurgical Societies.

predictive risk factors; and third, to justify our current policy of withholding anticonvulsants in the absence of seizures.

\section{METHODS}

\section{Patient population}

The study population was derived from the East Anglian regional audit of subarachnoid haemorrhage collected between 1994 and 2000, with a total affected population of 872. ${ }^{15}$ Outcome was independently assessed (by HMS) using the GOS six months after the event. The analysis was restricted to the first year post-bleed as the incidence of epilepsy has been shown to fall to very low levels after 12 months. ${ }^{16}$

The inclusion criterion was that the patient underwent a craniotomy to secure a ruptured intracranial aneurysm and survived to be discharged from acute neurosurgical care. Exclusion criteria are shown in table 1. Patients were managed according to standard protocols, which have been described in detail previously. ${ }^{15}$ Briefly, all patients were treated with fluid resuscitation (a combination of crystalloid and colloid, 3 litres/24 hours); good grade patients were treated with oral nimodipine (60 mg four hourly); poor grade patients were managed on the neuro-intensive care unit with placement of an external ventricular drain in the presence of hydrocephalus. Blood pressure was supported with inotropes if necessary and nimodipine was given through a centrally placed intravenous line ( $1-2 \mathrm{mg} /$ hour $)$, but reduced if there was a significant effect on blood pressure. A policy of early angiography and clipping within three days of haemorrhage was pursued for the good grade patients and for poor grade

Abbreviations: GOS, Glasgow outcome score; $\mathrm{SAH}$, subarachnoid haemorrhage; WFNS, World Federation of Neurosurgical Societies 
Table 2 Incidence of generalised epilepsy related to World Federation of Neurosurgical Societies grade, Glasgow outcome score, and site of aneurysm

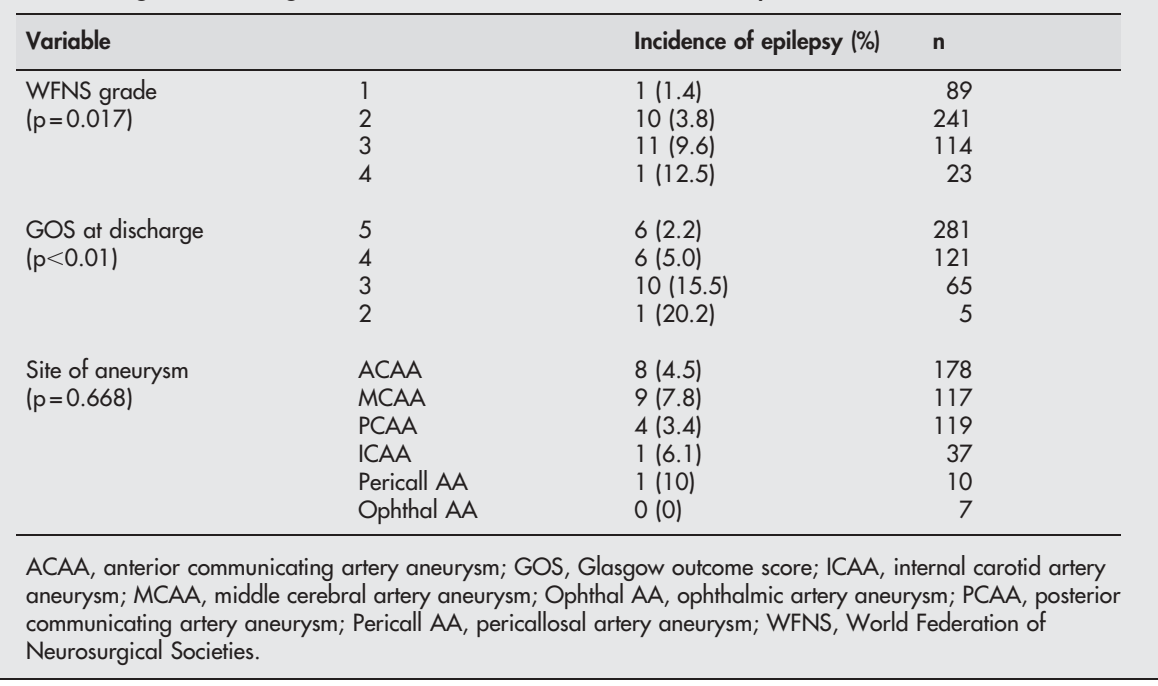

patients who had a motor score of 4 or more (flexion to pain or better) following reversal of sedation.

\section{Anticonvulsant treatment policy}

Prophylactic anticonvulsants were not prescribed in the absence of a perioperative seizure. If they were prescribed following an early seizure (within two weeks of the bleed) the patients were treated for three months. Treatment was continued for 24 months if a further seizure occurred.

\section{Data analysis}

Postoperative epilepsy and confounding risk factors were determined from the hospital medical records, including a dedicated prospectively designed proforma as part of the East Anglian regional audit of subarachnoid haemorrhage. This proforma includes demographic data, symptoms (with timings), past medical history, family history, drugs (long term and acute administration), systems review, GCS, the findings on neurological examination, World Federation of Neurosurgical Societies (WFNS) grading, general examination findings, and the results of all investigations (haematology and biochemistry, computed tomography, lumbar puncture, and angiography). Only convulsive seizures were included in the final analysis. The independent risk factors that were assessed were sex, WFNS grade on admission, discharge destination, site of aneurysm, and GOS at discharge, three months, and six months. The data were analysed using multivariate analysis (SPSS software).

\section{RESULTS}

Of the total study population in the audit $(n=872), 472$ fulfilled the criteria for inclusion. The mean age of patients was 53 years (range 15 to 84) and the male to female ratio was 1:2.01. The outcome for the patients at six months was good recovery in $81 \%$, moderate disability in $11 \%$, severe disability in $8 \%$, vegetative state in less than $0.1 \%$, and death in less than $0.1 \%$. Late epilepsy occurred in 23 patients $(4.9 \%)$. There was a significant relation between the incidence of late epilepsy and the presenting WFNS grade $(\mathrm{p}<0.05)$ (grade $1,1.4 \%$; grade $2,3.8 \%$; grade $3,9.6 \%$; grade $4,12.5 \%$ ) (table 2 ). There was also a significant relation between the incidence of late epilepsy and the GOS at discharge $(\mathrm{p}<0.01)$ (good recovery, $2.2 \%$; moderate disability, $5.0 \%$; severe disability, $15.5 \%$ ). There was no relation between the incidence of late epilepsy and sex or the site of the aneurysm.

\section{DISCUSSION}

In this study we aimed to determine the current risk for late epilepsy (more than two weeks after the event) following aneurysmal SAH treated by a modern regime involving early aneurysm surgery. ${ }^{15}$ The results show a low incidence of late epilepsy compared with other published series. ${ }^{38}$ Patients presenting with a poor grade-indicating a more severe primary insult-had a higher incidence of epilepsy (up to $20 \%$ ). This confirms that patients in worse condition before, during, and after surgery are more prone to developing epilepsy. The results do not support the view that there is a higher incidence of epilepsy in patients with middle artery aneurysms, ${ }^{10}$ and also showed no sex differences.

Although the East Anglian regional audit is prospective, the analysis was retrospective, with the risk that not all seizures were captured for the data analysis, and partial seizures were excluded. Furthermore, the use of exclusion criteria will inevitably lead to underestimation of the true overall frequency. Patients were also only followed for one year, so those developing epilepsy after that time were not included in the data analysis. This number is expected to be very small. ${ }^{16}$ Conversely, there was a chance of overreporting, with other neurological events being labelled as epilepsy because medical and nursing personnel rarely witness the seizures and there was no EEG evidence.

For good grade patients, the results support a policy of not prescribing prophylactic anticonvulsant drugs routinely, even for patients with middle cerebral artery aneurysms. For poor grade patients, the results are less clear. Adverse drug effects (often masked in such patients) and concerns over efficacy (which also apply to head injury) suggest that a policy of non-prescription is perhaps advisable. However, these patients should be monitored closely.

\section{Conclusions}

The study shows that in the modern era of aneurysm surgery, the overall incidence of late epilepsy is low and is significantly related to the Glasgow outcome score. Patients presenting with a poor grade have a higher incidence of epilepsy; these warrant further investigation in larger prospective studies to determine whether there is a role for anticonvulsants drugs in this population.

\section{ACKNOWLEDGEMENTS}

$\mathrm{PJH}$ is supported by a senior surgical scientist fellowship from the Academy of Medical Sciences/Health Foundation. 


\section{Authors' affiliations}

S J Buczacki, P J Kirkpatrick, H M Seeley, P J Hutchinson, Academic Department of Neurosurgery, University of Cambridge, Addenbrooke's Hospital, Cambridge, UK

Competing interests: none declared

Correspondence to: Mr P J Hutchinson, University of Cambridge Department of Neurosurgery, Box 167, Addenbrooke's Hospital Cambridge CB2 2QQ, UK; piah2@cam.ac.uk

Received 4 September 2003

Revised 4 February 2004

Accepted 10 February 2004

\section{REFERENCES}

1 Claassen J, Peery S, Kreiter KT, et al. Predictors and clinical impact of epilepsy after subarachnoid hemorrhage. Neurology 2003;60:208-14.

2 Ohman J. Hypertension as a risk factor for epilepsy after aneurysmal subarachnoid hemorrhage and surgery. Neurosurgery 1990;27:578-81.

3 Giroud M, Gras P, Fayolle H, et al. Early seizures after acute stroke: a study of 1640 cases. Epilepsia 1994;35:959-64.

4 Hasan D, Schonck RS, Avezaat CJ, et al. Epileptic seizures after subarachnoid hemorrhage. Ann Neurol 1993;33:286-91.
5 Ukkola V, Heikkinen ER. Epilepsy after operative treatment of ruptured cerebral aneurysms. Acta Neurochir (Wien) 1990; 106:115-18.

6 Keranen T, Tapaninaho A, Hernesniemi J, et al. Late epilepsy after aneurysm operations. Neurosurgery 1985;17:897-900.

7 Olafsson E, Gudmundsson G, Hauser WA. Risk of epilepsy in long-term survivors of surgery for aneurysmal subarachnoid haemorrhage: a population-based study in Iceland. Epilepsia 2000;41:1201-5.

8 Kotila M, Waltimo O. Epilepsy after stroke. Epilepsia 1992;33:495-8.

9 Bidzinski J, Marchel A, Sherif A. Risk of epilepsy after aneurysm operations. Acta Neurochir (Wien) 1992:119:49-52.

10 Shaw MD. Post-operative epilepsy and the efficacy of anticonvulsant therapy. Acta Neurochir Suppl (Wien) 1990;50:55-7.

11 Foy PM, Copeland GP, Shaw MDM. The incidence of post-operative seizures. Acta Neurochir 1981:55:253-64.

12 Johansson $M$, Cesarini KG, Contant CF, et al. Changes in intervention and outcome in elderly patients with subarachnoid hemorrhage. Stroke 2001;32:2845-949

13 Yap EC, Chua KS. Rehabilitation outcome after primary subarachnoid haemorrhage. Brain Ini 2002;16:491-9.

14 Osawa M, Hongo K, Tanaka Y, et al. Results of direct surgery for aneurysmal subarachnoid haemorrhage: outcome of 2055 patients who underwent direct aneurysm surgery and profile of ruptured intracranial aneurysms. Acta Neurochir (Wien) 2001;143:655-63.

15 Ross N, Hutchinson PJ, Seeley H, et al. Timing of surgery for supratentorial aneurysmal subarachnoid haemorrhage: report of a prospective study. J Neurol Neurosurg Psychiatry 2002;72:480-4.

16 Burn J, Dennis M, Bamford J, et al. Epileptic seizures after a first stroke: the Oxfordshire Community Stroke Project. BMJ 1997;315:1582-7.

\section{Call for papers}

10th European Forum on Quality Improvement in Health Care 13-15 April 2005, ExCel, Docklands, London

For further information on how to submit your paper please go to:

http://www.quality.bmipg.com 